\title{
DIE ENTWICKLUNG DES FINNISCHEN PFLANZENBAUS NACH DEM ZWEITEN WELTKRIEG
}

\author{
Otto VAlle
}

\begin{abstract}
Landwirtschaftliches Forschungsinstitut, Abteilung für Pflanzenbau, Tikkurila.
\end{abstract}
Während der zwei ersten Jahrzehnte der Selbständigkeit Finnlands in den 20er und 30er Jahren war die Entwicklung der finnischen Landwirtschaft günstig: das Ackerareal stieg durch Neukultivierung um ca. 600000 ha, und in bezug auf verschiedene landwirtschaftliche Produkte war Finnland Ende der 30er Jahre vor Ausbruch des zweiten Weltkrieges beinahe Selbstversorger. Im Produktionsjahr 1938/39 war Finnland bis zu 90 \% Selbstversorger in Brotgetreide und hatte einen bedeutenden Export-Ưberschuss an tierischen Produkten (Butter, Käse, Eier und Fleisch).

Nach dem zweiten Weltkrieg, während der letzten Hälfte der 40er Jahre, war Finnlands Landwirtschaft gelähmt. Das Land hatte beim Friedensschluss ca. 300000 ha Ackerland verloren, und die Kolonisation, die der Ansiedlung der ehemaligen karelischen Bevölkerung galt, erhöhte die Anzahl der Kleinbetriebe um mehrere Zehntausend. Verschiedene Hilfsmassnahmen, insonderheit für die Kleinbetriebe, waren notwendig. Finnlands Landwirtschaft hat nach dem zweiten Weltkrieg nicht dieselbe gesunde Entwicklung gehabt wie in den 30er Jahren.

Erfreulicherweise ist die Kultivierung neuen Ackerlandes nach dem Kriege bedeutend gewesen, denn schon im nächsten Jahr, 1958, wird die Ackerfläche gleich gross sein wie vor dem zweiten Weltkrieg, nämlich ca. 2.6 Mill. ha. Ackerbares Land gibt es am meisten in den nördlichen und östlichen Teilen Finnlands, und dort hat die Ackerfläche nach dem zweiten Weltkrieg erheblich zugenommen, z.B. im Län Lappland um ca. $66 \%$.

Der Anbau hat nach dem Krieg eine deutliche Intensivierung gezeigt. Der Handelsdüngerverbrauch ist bedeutend gestiegen im Vergleich mit den Jahren 1936 - 40: der Stickstoffverbrauch je ha hat sich nahezu versechsfacht, Phosphordünger verwendet man nun je ha $2.5 \mathrm{mal}$ und Kalidünger fast $4 \mathrm{mal}$ soviel als vor dem Kriege. Auch der Kalksteinmehlverbrauch beträgt nunmehr des Vierfache von dem des vorkriegszeitlichen. Es dürften nun für anspruchsvollere Gewächse und höhere Erträge bessere Möglichkeiten als früher bestehen.

Die Entwicklung nach dem Kriege und der Mangel an menschlicher Arbeitskraft in der Landwirtschaft haben die Mechanisierung auch in den Kleinbetrieben 
Tabelle 1. Die Nutzung der Ackerfläche in Finnland in den Jahren 1939 und 1956.

\begin{tabular}{|c|c|c|}
\hline Pflanzenart & $\begin{array}{c}1939 \\
\%\end{array}$ & $\begin{array}{c}1956 \\
\%\end{array}$ \\
\hline Winterweizen $\ldots \ldots \ldots \ldots \ldots \ldots$ & 1.0 & 0.9 \\
\hline Sommerweizen $\ldots \ldots \ldots \ldots \ldots \ldots$ & 4.2 & 4.2 \\
\hline Roggen $\ldots \ldots \ldots \ldots \ldots \ldots \ldots \ldots$ & 8.3 & 3.4 \\
\hline Gerste $\ldots \ldots \ldots \ldots \ldots \ldots \ldots \ldots \ldots$ & 4.5 & 7.4 \\
\hline Hafer $\ldots \ldots \ldots \ldots \ldots \ldots \ldots$ & 17.9 & 17.9 \\
\hline Gemenge $\ldots \ldots \ldots \ldots \ldots \ldots \ldots$ & 0.4 & 1.1 \\
\hline Erbsen $\quad \ldots \ldots \ldots \ldots \ldots \ldots \ldots \ldots$ & 0.5 & 0.4 \\
\hline Kleegras $\ldots \ldots \ldots \ldots \ldots \ldots \ldots$ & 44.5 & 44.9 \\
\hline Weidefutterflächen $\quad \ldots \ldots \ldots \ldots \ldots$ & 6.8 & 8.5 \\
\hline Gras- und Kleesamenbau $\ldots \ldots \ldots$ & 1.2 & 1.2 \\
\hline Grünfutter $\ldots \ldots \ldots \ldots \ldots \ldots \ldots$ & 0.7 & 1.0 \\
\hline Kartoffeln $\ldots \ldots \ldots \ldots \ldots \ldots \ldots$ & 3.4 & 3.6 \\
\hline Zuckerrüben $\ldots \ldots \ldots \ldots \ldots \ldots \ldots$ & 0.2 & 0.6 \\
\hline Sonstige Hackfrüchte $\ldots \ldots \ldots \ldots$ & 0.7 & 0.6 \\
\hline Markstammkohl $\quad \ldots \ldots \ldots \ldots \ldots \ldots$ & 0.1 & - \\
\hline Blattrüben $\ldots \ldots \ldots \ldots \ldots \ldots \ldots$ & - & 0.3 \\
\hline Faserpflanzen $\quad \ldots \ldots \ldots \ldots \ldots \ldots$ & 0.1 & - \\
\hline Oelfrüchte $\ldots \ldots \ldots \ldots \ldots \ldots \ldots$ & - & 0.5 \\
\hline$\ldots \ldots \ldots \ldots \ldots \ldots \ldots \ldots$ & 5.2 & 2.6 \\
\hline \multirow[t]{3}{*}{ Sonstige Flächen $\ldots \ldots \ldots \ldots \ldots$} & 0.3 & 0.9 \\
\hline & 100.0 & 100.0 \\
\hline & 2.63 & 2.59 \\
\hline
\end{tabular}

beschleunigt. In den letzten 15 Jahren ist die Traktorenzahl in der Landwirtschaft von 6000 auf 53000 angestiegen. Der Mähdrescher war noch um 1950 in Finnland beinahe unbekannt, aber nun ist die Anzahl der Mähdrescher bis auf über 3000 angestiegen.

Wenn man den derzeitigen Pflanzenbau in Finnland mit dem der Vorkriegszeit vergleicht, sind viele bedeutende Veränderungen zu beobachten (Tab. 1). Die am meisten in die Augen fallende und betrüblichste Entwicklung ist der starke Rückgang des Brotgetreidebaus, der auf mancherlei Umstände zurückzuführen ist: u.a. die Preispolitik, unvorteilhafte Witterungsverhältnisse mit später Aussaat, Frost, Regen während der Erntezeit sowie schlechte Überwinterung des Wintergetreides.

Der Roggen war noch im Beginn der 40er Jahre die wichtigste Brotgetreideart in Finnland. Im Jahre 1939 hat der Roggenbau 218000 ha ( $8.3 \%$ der Ackerfläche) betragen, aber seitdem Jahr für Jahr abgenommen. In den vier letzten Jahren (1953-56) belief sich die Roggenfläche auf nur ca. 83000 ha oder ca. $40 \%$, verglichen mit der Vorkriegszeit Ende der 30er Jahre. In den 40er Jahren sank die Roggenfläche in dem gleichen Masse, wie die Sommerweizenfläche zunahm, aber später, in den 50er Jahren, trat ein starker Rückgang sowohl im Roggen- wie im Sommerweizenbau ein. Eine natürliche Ursache für die Verminderung des Roggenbaus war die Entwicklung, die die Bedeutung des Weizens als Brotgetreide auf Kosten des Roggens vergrösserte. Vor der Ausdehnung des Weizenbaus in Finn- 
land konsumierte man je Person und Jahr z.B. im Produktionsjahr 1928/29 $79 \mathrm{~kg}$ Roggen und $38 \mathrm{~kg}$ Weizen (68\% Roggen und $32 \%$ Weizen), in den 50er Jahren, als sich die Bedeutung des Brotes in der Nahrung verringerte, betrug der Anteil des Weizens am Brotgetreide $60 \%$ und der des Roggens nur $40 \%$. Der Rückgang des Roggenbaus ist so stark gewesen, dass der hiesige Anbau nicht dem Bedarf entspricht: im Produktionsjahr 1938/39 war Finnland in Roggen zu $97 \%$ Selbstversorger, aber $1955 / 56$ nur zu $40 \%$. Finnland hat einen Importbedarf an Roggen von ca. 100 Mill. kg und könnte daher seinen jetzigen Roggenbau um ca. 70000 ha vermehren.

Eine Ursache dessen, dass der Roggenbau so stark zurückgegangen ist, sind geringere Eignung und Anpassung des Winterroggens an den heutigen Pflanzenbau. Da die Überwinterungsverhältnisse in Finnland schwierig sind, ist man genötigt, fast ausschliesslich finnische Roggensorten zu bauen, die im Stroh lang sind. Die schwedischen oder deutschen kurzstrohigen Roggensorten sind nicht einmal für das südlichste Finnland ausreichend winterfest.

Während einer längeren Reihe von Jahren waren die Winter für die Überwinterung des Roggens schwer gewesen und die Erträge gering geblieben. Das lange Stroh des Roggens erhöht die Erntekosten im Vergleich zu Sommerweizen. Wegen des langen Strohes und der allgemeinen Lagerung verursacht auch der Mähdrusch des Roggens, im Vergleich mit anderen Getreidearten, Schwierigkeiten und Extrakosten. Auch der Auswuchsschaden ist in regnerischen Erntezeiten bedeutend. Auf der anderen Seite gleicht der herbstgesäte und zeitig reifende Roggen die Arbeitsspitzen sowohl im Frühjahr als auch im Herbst etwas aus. Möglich ist, dass sich in Zukunft durch Neuzüchtung anbauwertere Roggensorten, als das jetzige finnische Anbaumaterial darstellt, erzielen lassen.

An Weizen baut man augenblicklich ca. $80 \%$ Sommerweizen und $20 \%$ Winterweizen. Winterweizen kann vor allem auf den Tonböden Südfinnlands gebaut werden. Da der Winterweizen zeitiger reift als der Sommerweizen und sich gut für Mähdrusch eignet, besteht grosses Interesse für die Ausbreitung des Winterweizenbaus. Leider gibt es in Finnland nicht hinreichend anbauwerte Sorten. Der Mangel an neuen Zuchtsorten macht sich bei Winterweizen stärker bemerkbar als bei den anderen Getreidearten. Die neuesten finnischen Winterweizensorten (V a k k a, A n t t i) zeigen in regnerischen Herbsten Neigung zu Auswuchs auf dem Felde. Die Anbaufläche für Winterweizen könnte leicht auf ca. 50000 ha verdoppelt werden, wenn anbauwerte, sichere Sorten auf den Markt gebracht werden könnten.

Als der Sommerweizenbau im Beginn der 30er Jahre zuzunehmen begann, erweiterte sich die Anbaufläche fortgesetzt bis zum Jahre 1949, als man auf ganzen 181000 ha Sommerweizen baute. Seitdem ist die Sommerweizenfläche auf 104000 ha gesunken (1954). Die Ursache dieses schnellen und starken Rückgangs war die Witterung, die besonders in den 50er Jahren von den günstigen 30er Jahren abwich. Frost vor der Reife verursachte das Schwinden des Sommerweizens in den nördlichsten sommerweizenbauenden Gegenden. Reichliche Niederschläge während mehrerer Herbste erschwerte die Erntearbeit und verschlechterte die Qualität. Einige ungewöhnlich späte Früjahre (1955 und 1956) haben ebenfalls das Interesse der Landwirte für Sommerweizen verringert. 
Da Finnland gegenwärtig nur zu $40 \%$ Selbstversorger in Weizen ist und einen jährlichen Importbedarf von 250 Mill. kg Weizen hat, bestehen Möglichkeiten, den Weizenbau und besonders den Sommerweizenbau von neuem auszudehnen, soweit eben die Wetterverhältnisse in den nächsten Jahren günstig sind. Beim Weizen erstrebt man in Finnland nur eine $75 \%$ ige Selbstversorung, um durch den Import der restlichen $25 \%$ die Qualität des Mehles auch nach ungünstigen Sommern aufrechterhalten zu können. Eine Vermehrung der Weizenbaufläche um ca. 100000 ha wäre wünschenswert. Durch Pflanzenzucht kann noch wertvolleres Anbaumaterial zustande gebracht werden. Bisher sind Svalöfs D i a m a n t- und D i a$\mathrm{m}$ a n t I I-Weizen im finnischen Weizenbau von entscheidender Bedeutung gewesen, aber für Mähdruschzwecke sind frühreifere Sorten mit guter Standfestigkeit und Qualität wünschenswert. Unter den frühreifen Sorten besteht für A p u-Weizen, von der Pflanzenzuchtanstalt Jokioinen, grösstes Interesse, aber dieser ist schwach im Stroh, anfällig gegen Flugbrand und qualitativ nicht ebenso gut wie die besten Sommerweizensorten.

Der Gerstenbau hat während der letzten Jahre grossen Veränderungen unterstanden, denn seit 1950 ist die Gerstenfläche um ca. 80 \% gestiegen. Das hat, gegenüber anderen Getreidearten, an dem sicheren Reifen der Gerste in kurzen oder kühlen Sommern gelegen. Als frühreifende Frucht hat sich die Gerste auch als für Mähdrusch geeignet erwiesen. Sie hat eine vielseitige Verwendbarkeit, und zwar in der menschlichen Ernährung (ca. 60 Mill. kg), in der Malzindustrie (ca. 18 Mill. kg) und als Futter.

Man baut in Finnland in den südlichen Teilen des Landes zweizeilige Gerste, sechszeilige wiederum in Mittel- und Nordfinnland. Die wertvollste zweizeilige Gerstensorte ist heuzutage Weibullsholms B a l d e r, die sowohl standfest als auch ertragsreich ist. Jedoch zeigt die Gerste in regnerischen Ernteperioden Neigung zu Auswuchs, weshalb man für spät keimreifende Sorten Interesse hat. Eine vorzügliche Braugerstensorte soll die dänische $\mathrm{Carlsberg}$ I I sein.

Bisher ist in Finnland nur zweizeilige Gerste als Braugerste genehmigt worden, aber schon im Herbst 1956 hat man eine sechszeilige Gerste, Tammistos P i r k k a, als Braugerste zugelassen. Pirkka reift ca. 10 Tage früher als Balder und wird den Braugerstenbau in Finnland sicherstellen. Im Sommer 1956 baute man Gerste auf 192000 ha, d.h. $7.4 \%$ der Ackerfläche.

Der Hafer ist in Finnland schon lange die am meisten gebaute Getreideart und hat eine ebenso weite Verbreitung wie Roggen, Weizen und Gerste zusammen. Am meisten baut man Weisshafer; Schwarzhafer jedoch auch, vor allem in Nordfinnland. Hafer ist das wichtigste Futterkorn, nur ca. 60 Mill. $\mathrm{kg}$ (unter $10 \%$ des Ertrages) verwendet man als Graupenhafer. In Finnland gibt es viele wertvolle finniche Haferzüchtungen, so z.B. Tammistos S i s u, E h o und T a m mi. Svalöfs Goldreg e n I I ist im Schwinden begriffen, da er sehr schwach im Stroh ist. Von den neuen Sorten ist der holländische, standfeste P e n d e k-Hafer auf humusreichen Böden sehr ertragsreich und eignet sich gut als Decksaat. Er wurde in Finnland im Frühjahr 1957 auf den Markt gebracht.

In den Jahren 1950 und 1955 hat man in Finnland Untersuchungen durchgeführt, die der Verbreitung der verschiedenen Zuchtsorten im Lande galten. Die 
Tabelle 2. Die Verbreitung der wichtigsten Getreide- und Erbsensorten in Finnland in den Jahren 1950 und 1955.

Anteil der verschiedenen Sorten in Prozent von der Anbaufläche jeder Art.

$\mathrm{T}=$ Tammisto, $\mathrm{J}=$ Jokioinen, $\mathrm{S}=$ Svalöv, $\mathrm{W}=$ Weibullsholm, $\mathrm{D}=$ Dänemark, $\mathrm{E}=$ Estland

Winter weizen

$\begin{array}{cccccc}\text { Jahr } & \text { Varma } & \text { Olympia } & \text { Virtus } & \text { Vakka } & \text { Andere } \\ & \mathrm{T} & \mathrm{J} & \mathrm{W} & \mathrm{J} & \text { Sorten } \\ 1950 & 64.2 & 20.9 & 0.5 & - & 14.4 \\ 1955 & 56.8 & 10.2 & 8.7 & 4.4 & 19.9\end{array}$

Winterroggen

$\begin{array}{cccccccc}\text { Jahr } & \text { Toivo } & \text { Landsorten } & \text { Ensi } & \text { Pekka } & \text { Sangaste } & \text { Oiva } & \text { Andere } \\ & \mathrm{J} & & \mathrm{J} & \mathrm{J} & \mathrm{E} & \mathrm{T} & \text { Sorten } \\ 1950 & 25.3 & 26.5 & 10.5 & 6.2 & 13.8 & 4.3 & 13.4 \\ 1955 & 21.1 & 18.0 & 16.9 & 13.3 & 12.8 & 4.2 & 13.7\end{array}$

So $m$ m er weize n

Jahr

1950

1955

Jahr

1950

1955

Jahr

1950

1955

Jahr

1950

1955

$\begin{array}{cc}\text { Diamant } & \text { Diamant II } \\ \text { S } & \text { S } \\ 60.0 & 22.8 \\ 27.8 & 25.0\end{array}$

Kärn I

W

2.5

12.1

Apu

Tammi

Terä

$\mathrm{T}$ T

6.7

10.8

3.5

Gerste

$\begin{array}{cccccc}\text { Tammi } & \text { Balder } & \text { Binder } & \text { Vega } & \text { Pirkka } & \text { Andere } \\ \text { T } & \text { W } & \text { D } & \text { S } & \text { T } & \text { Sorten } \\ 15.9 & 12.7 & 33.9 & 23.4 & - & 14.1 \\ 36.6 & 25.5 & 15.0 & 9.8 & 5.1 & 8.0\end{array}$

II a f e r

$\begin{array}{cc}\text { Sisu } & \text { Tammi } \\ \mathrm{T} & \mathrm{T} \\ 10.6 & 14.9 \\ 29.1 & 23.2\end{array}$

Eho

$\mathrm{T}$

12.1

16.1

Goldre

$$
\mathrm{S}
$$

42.9

15.5

Erbse

$\begin{array}{cccccc}\text { Torsdag II } & \text { Sinikka } & \text { Kalle } & \text { Concordia } & \text { Martta } & \text { Andere } \\ \text { S } & \text { J } & \text { T } & \text { S } & \text { T } & \text { Sorten } \\ 37.4 & 17.9 & - & 8.5 & 6.7 & 29.5 \\ 8.9 & 14.0 & 63.6 & 2.5 & 1.7 & 9.3\end{array}$

\section{Andere Sorten \\ 8.0 \\ 9.9}


Zusammenfassung über dieses Material ist in der Tabelle $2 \mathrm{zu}$ sehen. Dieser Berechnung nach baute man im Jahre 1955 an Getreide und Erbsen über die Hälfte, $52 \%$, Tammisto-Züchtungen und Svalövs Sorten zu $23 \%$. Die staatliche Pflanzenzüchtungsanstalt in Jokioinen ist mit $8 \%$ und Weibullsholm mit $7 \%$ vertreten. Die finnischen Züchtungen haben mit der Zeit immer grössere Bedeutung erlangt.

Im Futterbau sind im letzten Jahrzehnt keine grösseren Veränderungen zu beobachten. Der Kleegrasbau basiert seit Jahrzehnten auf Rotklee, Schwedenklee und Timothee. In Südfinnland sind die Futterbauflächen kleereicher als in Nordfinnland. Der Mangel an Rotkleesamen hat die Verjüngung der Kleegrasflächen und die Zunahme des Kleegehaltes auf den Futterflächen verzögert. Da der Rotklee Finnlands wertvollste Futterleguminose ist, sucht man mit verschiedenen anbautechnischen Massnahmen das Gedeihen des Rotklees und seine Ausdauer zu fördern. Das Rotkleesamenproblem in Finnland wartet auf seine Lösung.

Die grösste Veränderung im Futterbau erfuhren in der Nachkriegszeit die Weidefutterflächen. Ein grosser Teil der Weidefutterflächen auf Ackerland sind noch alte Kleegrasschläge, die geringe Erträge geben. Man beginnt zur zeit ein immer grösseres Interesse für sog. kurzzeitige Weidefutterflächen zu zeigen, in denen die dominierende Grasart entweder Wiesenschwingel (Festuca pratensis) oder Knaulgras (Dactylis glomerata) ist. Durch starke jährliche Düngung erhält man diese Futterflächen etwa sechs Jahre produktiv. Der Hauptteil des Wiesenschwingel- und Knaulgrassamens wird aus Dänemark importiert.

Neben den mehrjährigen Kleegras- und Grasflächen besteht Bedarf an einjährigen Futterpflanzen, insonderheit in Nordfinnland. Nach dem Kriege hat die sog. Blattrübe, eine blattreiche Form der Wasserrübe, als Rohstoff sowohl für AIV-Futter wie auch als Zusatzfutter während oder nach der Weidezeit Bedeutung gewonnen. Die Blattrübe baut man gegenwärtig auf ca. 7000 ha. Da sich die Kohlfliege im Blattrübenbau als lästig erwiesen hat, so hat man in den letzten Jahren, besonders in Nordfinnland, Sommerraps und Winterraps als Futterpflanze anzubauen begonnen. Markstammkohl hat leider nach dem Kriege nicht mehr dieselbe Bedeutung wie in den 30er Jahren.

Der Futter-Hackfruchtbau ist in Finnland in den letzten Jahrzehnten stark zurückgegangen. Noch 1930 baute man Turnips auf 23000 ha an, aber die Anbaufläche sank 1939 auf 9000 ha und beträgt nun nur noch ca. 2000 ha. Kohlrüben baut man jetzt auf ca. 4000 ha und Futterrüben sowie Futterzuckerrüben auf ca. 3000 ha. Wenn man den Futter-Hackfruchtbau durch mechanisiertes Pflanzen und Ernten anregen könnte, würden in Finnland die Futterzuckerrüben die wertvollsten Futter-Hackfruchtarten sein.

Als Industriepflanzen baut man in Finnland sowohl Zuckerrüben als auch Speiseölfrüchte. Die erste Zuckerfabrik begann ihre Tätigkeit 1919 in Salo. Augenblicklich gibt es im Lande fünf Fabriken, von denen drei erst seit einigen Jahren tätig gewesen sind. Finnland ist das nördlichste Land der Welt, das Zuckerrüben baut, und darum begegnet der Anbau vielen Schwierigkeiten infolge der kurzen Vegetationsperiode und der oftmals unvorteilhaften Witterungsverhältnisse. Die Erträge sind bisher gering (ca. $20000 \mathrm{~kg} / \mathrm{ha}$ ) und ebenfalls der Zuckergehalt niedriger 


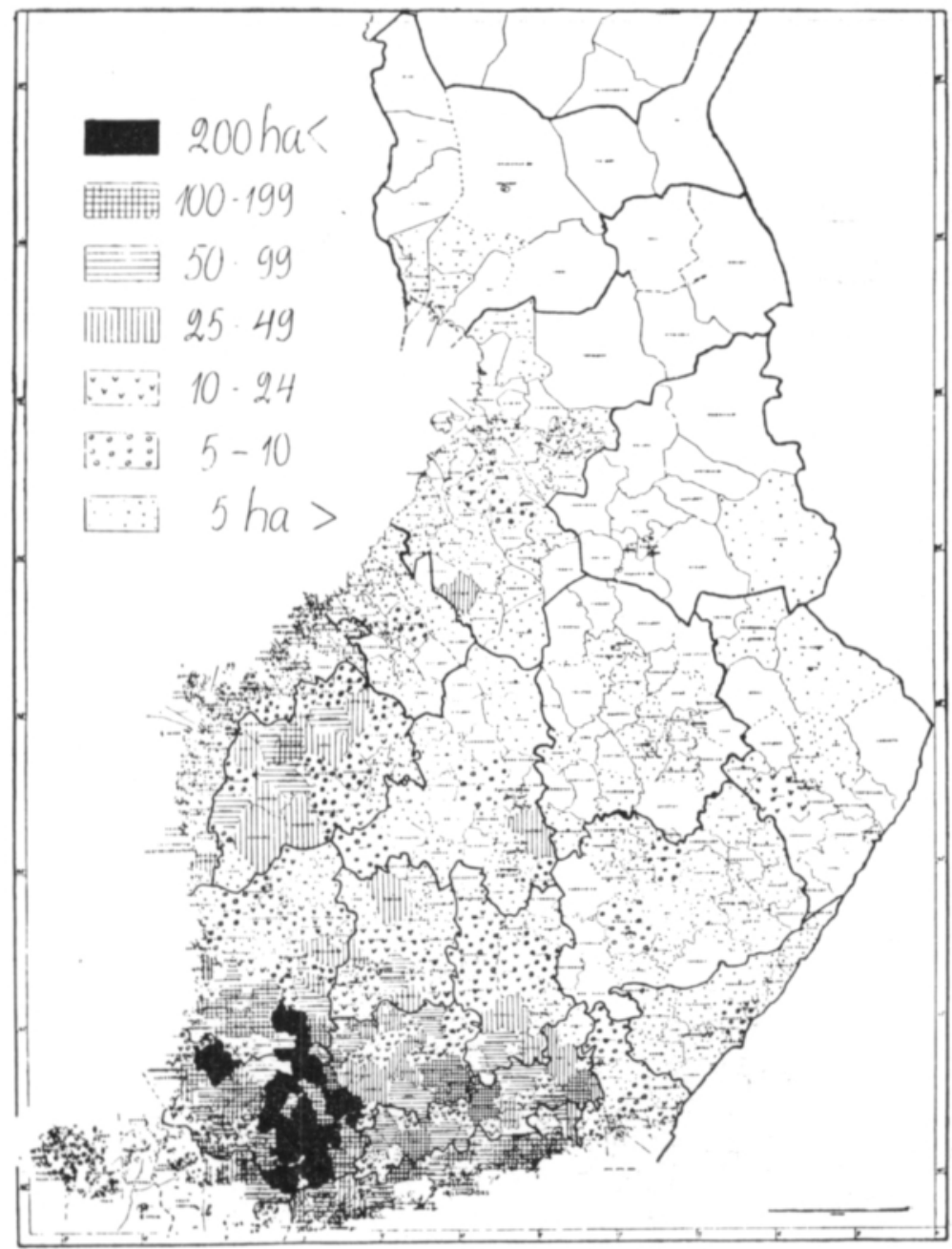

Abb. 1. Die Anbaufläche von Winterrübsen, gesät im Jahre 1953, in Finnland. Gesamtfläche 17.250 ha. Die Abbildung zeigt die Anbaufläche von Winterrübsen in den verschiedenen Kirchspielen. Das wichtigste Anbaugebiet liegt in Südwestfinnland. In schneereichen Gegenden im inneren und östlichen Finnland ist der Anbau von Winterrübsen gering.

als in den südlicheren Ländern gewesen. Die Zuckerrübenfläche beträgt, aus Mangel an Anbauern, nur ca. 15000 ha (1956) und wäre auf mindestens 20000 ha zu erhöhen. Die Zuckerrübe ist eine wertvolle Vorfrucht für mehrere andere Feldfrüchte. An Speiseölfrüchten baut man in Finnland nur Winterrübsen, und zwar ca. 15000 ha. Dieser Anbau begann nach dem Kriege, Ende der 40er Jahre. Das Zentrum des Winterrübsenbaus liegt in Südwestfinnland, wo man auch eine Ölraffinerie gebaut hat. Winterrübsen wird überall in Südfinnland gebaut, ferner auf kleineren Flächen noch ziemlich weit im Norden, in den schneearmen westlichen Teilen des Landes bis nach Nord-Ostbottnien, bei c. $64^{\circ} \mathrm{n}$. Br. Durch den Winterrübsenbau kann man ca. 1/3 des Margarineöls im eigenen Lande erzeugen. Der Extraktionsrest, 


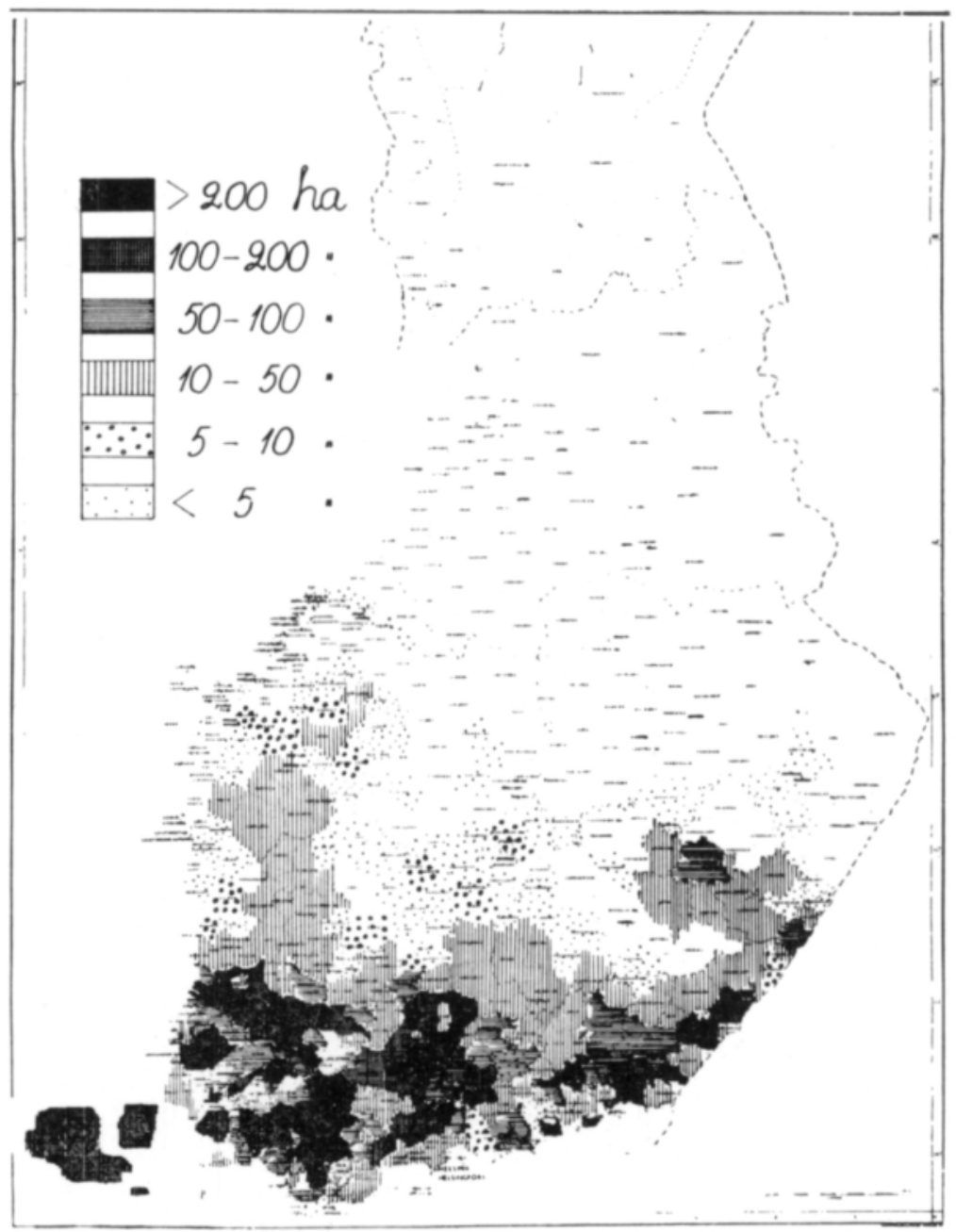

Abb. 2. Die Anbaufläche von Zuckerrüben in Finnland im Jahre 1955. Gesamtfläche c. 17.000 ha. In Finnland gibt es 5 Rübenzuckerfabriken, alle in Südfinnland. Die Abbildung zeigt die Anbaufläche von Zuckerrüben in den verschiedenen Kirchspielen.

das Rübsenschrot, ist als Ölkraftfutter ebenfalls von Wert. Der Winterrübsen ist wegen seiner frühen Reife und seines hohen Vorfruchtwertes eine wertvolle Feldfrucht.

Im Obigen ist die Entwicklung des Pflanzenbaus in Finnland nach dem zweiten Weltkrieg kurz geschildert worden. Trotz dem intensiveren Anbau, im Vergleich mit den 30er Jahren, sind die ha-Erträge jedoch nicht, wie man infolge der verbesserten Düngung hätte annehmen können, gestiegen. Das beruht wahrscheinlich auf den verschlechterten Witterungsverhältnissen insbesondere während der 50er Jahre. Die 30er Jahre waren ein ausnahmsweise warmes und für den Pflanzenbau günstiges Jahrzehnt. In den 40er und besonders in den 50er Jahren gab es wiederholt viele ungünstige Sommer, die der Landwirtschaft grossen Schaden zufügten. In 
den 50er Jahren kamen schon zwei ungewöhnlich späte Frühjahre, drei regnerische und vor allem für den Mähdrusch beschwerliche Herbste sowie zwei kalte Sommer mit mangelhafter Reife vor. Im Herbst 1956 traten vor dem Ausreifen des Sommergetreides in fast ganz Finnland äusserst schwere Nachtfröste auf, weshalb der Importbedarf allein an Hafer im Frühjahr 1957 ca. 20 Mill. kg ausmachte. Diese für den Pflanzenbau ungünstigen Sommer haben anderseits die Vermehrung der viehlosen Betriebe aufgehalten.

Wenn die nächsten Jahre in ihren Wärme- und Regenverhältnissen annähernd normal werden, so wird der Brotgetreidebau schnell und stark zunehmen. Auch stehen grosse Aufgaben zugunsten der Förderung des finnischen Futterpflanzenbaus bevor, denn der Kraftfutterimport hat sich während der letzten Jahre im Vergleich mit dem der Vorkriegszeit beinahe verdoppelt. Um den Pflanzenbau zu rationalisieren, ist es auch notwendig, die Dränung zu beschleunigen. Die in Finnland allgemeinen offenen Gräben erschweren die Mechanisierung des Pflanzenbaus und somit die Rationalisierung der gesamten Betriebe.

Auch die Forschung hat im Hinblick auf die Förderung des finnischen Pflanzenbaus bedeutungsvolle Aufgaben zu erfüllen, um das Streben der Landwirte nach höheren und qualitativ besseren Erträgen zu erleichtern. In Finnland wurde vom Beginn des Jahres 1957 an das gesamte Versuchswesen umorganisiert, um die Forschungsarbeit zu intensivieren sowie eine engere Zusammenarbeit zwischen den Forschern und einen engeren Kontakt zwischen Forschung und Beratung zustande zu bringen. Neue Probleme auch innerhalb des Pflanzenbaus tauchen jedes Jahr in der Praxis auf. $\mathrm{Zu}$ ihrer schnellen Lösung bedarf es mehr denn je der Zusammenarbeit zwischen den einzelnen Forschungsinstituten einerseits sowie zwischen den Vertretern von Praxis und Forschung anderseits.

SELOSTUS:

SUOMEN KASVINTUOTANNON KEHITYS TOISEN MAALMANSODAN JÄLKEEN

OtTo VALLE

Maatalouden tutkimuskeskus, Kasvinviljelylaitos, Tikkurila

Toisen maailmansodan jälkeen on Suomen kasvintuotanto ollut merkittävien muutosten alainen (taul. 1). Maan leipăviljaomavaraisuus on huomattavasti heikentynyt erityisesti 1950-luvulla ja sekä rukiin ettă vehnän tuontitarve on lisääntynyt. Jotta Suomi olisi rukiissa omavarainen, voitaisiin rukiin viljelyalaa lisătä n. 70000 ha:lla. Jotta taas vehnässä saavutettaisiin $75 \%$ omavaraisuus, voitaisiin vehnän viljelyalaa lisätä n. 100000 ha:lla.

Ohran viljelyala on 1950-luvulla voimakkaasti kasvanut johtuen sen aikaisesta tuleentumisesta ja viljelyvarmuudesta muihin kevätviljoihin verrattuna. Kauran viljelylaajuus on ollut sama kuin muiden viljalajien yhteensä.

Vuosina 1950 ja 1955 on selvitetty eri vilja- ja hernelajikkeiden levinneisyyttä Suomessa (taul. 2). 
Vilja- ja hernealasta oli 1955 Tammiston jalosteiden kasvussa $52 \%$, Svalövin jalosteiden osuus oli $23 \%$, Jokioisten $8 \%$ ja Weibullsholmin $7 \%$.

Rehukasvien viljelyssä ei ole tapahtunut merkittäviä muutoksia. Tärkeimmän palkokasvin, puna-apilan, siementuotannossa on ollut suuria vaikeuksia. Laidunheinistä on nurminadan ja koiranheinän merkitys lisääntynyt.

Teollisuuskasveista on sekä syysrypsiä että sokerijuurikasta viljelty n. 17000 ha:n alalla (kuva 1 ja 2). 